\title{
Correction to: Influence of time interval on the water entry of two spheres in tandem configuration
}

\author{
Xujian Lyu ${ }^{1} \cdot$ Honglu Yun ${ }^{1,2} \cdot$ Zhaoyu Wei $^{2} \mathbb{D}$
}

Published online: 29 November 2021

๑) Springer-Verlag GmbH Germany, part of Springer Nature 2021

\section{Correction to: Experiments in Fluids (2021) 62:222 https://doi.org/10.1007/s00348-021-03300-w}

After publication of the original manuscript, it was realized that the work of Rabbi et al. (2021) was not properly referenced. Therefore, the specific reference is listed herein, and the following changes are recommended:

1. The sentence in the abstract of "The result shows that decay rate of acceleration for the second sphere decreases with increasing $M t$." should be replaced with "The result shows that the decay rate of acceleration for the second sphere generally decreases with increasing $M t$, beyond the value of $M t$ investigated in past studies $(M t>6)$."

2. The sentence in section 4.2 of "..., close to the critical value mentioned by Rabbi et al. (2021) for the water entry of multi-droplet streams." should be changed to "..., close to the critical value mentioned by Rabbi et al. (2021) for the water entry of consecutive spheres."

3. The reference of Rabbi et al. (2021) should be added to the last paragraph of the section "2. Experimental methods" and also to the last paragraph of the introduction, respectively, as:

"The Matryoshka number is a dimensionless parameter defined by the ratio of time interval and pinch-off time as $M t$ $=\Delta t / t_{\mathrm{p}}($ Rabbi et al. 2021)."

"What's more, Yun et al. (2020a, b) and Rabbi et al. (2021) have conducted some preliminary studies on the cavity dynamics and motion characteristic of two spheres

The original article can be found online at https://doi.org/10.1007/ s00348-021-03300-w.

Zhaoyu Wei

wzhyu@sjtu.edu.cn

1 School of Energy and Power Engineering, Nanjing University of Science and Technology, Nanjing 210094, People's Republic of China

2 School of Oceanography, Shanghai Jiao Tong University, Shanghai 200240, People's Republic of China entering into water in tandem configuration with different initial velocities and found that the cavity evolution is completely different as compared to that of single sphere."

\section{Reference}

Rabbi R, Speirs N, Kiyama A, Belden J, Truscott T (2021) Impact force reduction by consecutive water entry of spheres. J Fluid Mech 915:A55. https://doi.org/10.1017/jfm.2020.1165

Publisher's Note Springer Nature remains neutral with regard to jurisdictional claims in published maps and institutional affiliations. 